\title{
Combined titre and fluorescent pattern of IgG antinuclear antibodies using cultured cell monolayers in evaluating connective tissue diseases
}

\author{
MICHAEL D. PARKER AND GRACE P. KERBY \\ From the Rheumatic Disease Section of the Department of Medicine, Duke University Medical Center, \\ Durham, North Carolina
}

After the introduction of immunofluorescence in 1957 as a technique to detect antinuclear antibodies (Friou, 1957; Holborow, Weir, and Johnson, 1957), it was reported that with rare exception these antibodies are found in patients with systemic lupus erythematosus (SLE) (Schur, 1970; Gonzalez and Rothfield, 1966). Antinuclear antibodies (ANA) are also found frequently in other connective tissue diseases (Ritchie, 1970; Rothfield and Rodnan, 1968; Beck, Anderson, Bloch, Buchanan, and Bunin, 1965; Alexander, Bremner, and Duthie, 1960; Sharp, Irvin, Tan, Gould, and Holman, 1972; Friou, 1967; Ruderman, Miller, and Pinals, 1968) and less frequently in a variety of illnesses (Alexander and others, 1960). Several approaches have been used to differentiate certain connective tissue diseases serologically on the basis of ANA specificity or titre (Sharp and others, 1972; Pincus, Schur, Rose, Decker, and Talal, 1969; Ritchie, 1967; Northway and Tan, 1972). Particular ANA patterns of nuclear fluorescence (homogeneous, speckled, nucleolar, and peripheral) have been reported to be closely associated with specific connective tissue diseases (Beck, 1963; Gonzalez and Rothfield, 1966; Ritchie, 1970; Friou, 1967; Casals, Friou, and Myers, 1964). However, the potential clinical value of ANA patterns is not clearly established due to conflicting reports concerning the disease specificity of each pattern (Gonzalez and Rothfield, 1966; Ritchie, 1970; Rothfield and Rodnan, 1968; Northway and Tan, 1972; Dorsch, Gibbs, Stevens, and Shulman, 1969).

The present study was undertaken for the dual purpose of assessing the value of (1) using cultured Vero cells in monolayer as a uniform substrate yielding more easily interpreted fluorescent ANA patterns, and (2) combining determination of ANA titres and fluorescent patterns to increase the diagnostic specificity of serological data in connective tissue diseases. The fluorescent patterns obtained with Vero cells were more easily interpreted than those with cryostat sectioned tissues. Combined determination of ANA titres and fluorescent patterns did provide more useful information in the serological study of SLE, scleroderma, and mixed connective tissue disease (MCTD) than did either test alone. Neither test, alone or in combination, was useful in a positive sense in the evaluation of other connective tissue diseases studied.

\section{Methods}

PATIENTS (Parker, 1973)

Normal 162. Patients with illnesses other than connective tissue diseases 143. Patients meeting A.R.A. criteria for definite or classical rheumatoid arthritis (Ropes, Bennett, Cobb, Jacox, and Jessar, 1958) 119. Patients with SLE by A.R.A. criteria (Cohen, Reynolds, Franklin, Kulka, Ropes, Shulman, and Wallace, 1971) 66. Seven had mild myositis and 2 had sclerodactyly. Patients with polymyositis 9. Two had sclerodactyly. Patients with scleroderma 18. One had a Coomb's positive haemolytic anaemia and a prior episode of myositis and another had persistently raised muscle enzymes without weakness. Patients with mixed connective tissue disease with clinical features as previously reported (Parker, 1973) 10. Patients with other connective tissue diseases 14: 2 Sjögren's syndrome, 3 juvenile rheumatoid arthritis, 1 widespread vasculitis, 1 Felty's syndrome, 3 ankylosing spondylitis, 3 psoriatic arthritis, and 1 eosinophilia and arthritis.

Criteria used here for the diagnosis of scleroderma, polymyositis, and MCTD have been reported previously (Parker, 1973). 
FLUORESCENT METHODS

Nuclear substrates were prepared by the methods of Rothfield, Frangione, and Franklin (1965) (mouse liver) and Tan (1967) (mouse kidney). In addition, coverslip cultures of African green monkey kidney cells (Vero*) were grown in Leighton tubes in Dulbecco's medium plus $10 \%$ fetal calf serum. Cells were fixed in methanol, dried, and stored in ointment jars with dessicant at $-20^{\circ} \mathrm{F}$ for up to 12 weeks without change in pattern or titre of nuclear fluorescence. Commerical fluorescein-conjugated goat antisera to the heavy chains of human IgG and IgM were purchased from Hyland Laboratories. Each lot was checked by methods previously described (Parker, 1973) to ensure that the antibody was monospecific to either IgG or IgM. Fluorescent staining for each nuclear substrate was carried out by the techniques recommended by the respective authors (Rothfield and others, 1965; Tan, 1967). Vero cell coverslip cultures were placed on rubber stoppers in moisture chambers, covered with $0.1 \mathrm{ml}$ of appropriately diluted serum, and incubated at $37^{\circ} \mathrm{C}$ for 30 mins. Coverslips were then washed for 10 mins in $0.01 \mathrm{~mol} / 1$. phosphate-buffered saline $\mathrm{pH} 7.4$ (PBS) and returned to the chamber. $0.1 \mathrm{ml}$ of a $1: 1$ mixture of $1 \mathrm{mg} / 100 \mathrm{ml}$ Evans blue in PBS and appropriately diluted fluorescein-conjugated antiserum to either IgG or IgM was applied to each coverslip and then incubated another $30 \mathrm{mins}$ at $37^{\circ} \mathrm{C}$. Finally, the coverslips were washed for 10 mins, mounted on a microscope slide, and sealed with clear fingernail varnish. In some studies, nuclear substrates were treated with deoxyribonuclease or ribonuclease (Worthington) as described by Sharp and others (1972) before being used in the test. Titrations of serum samples were done by making serial fourfold dilutions beginning with a $1: 10$ dilution.

\section{PRECIPITIN REACTIONS}

Soluble nuclear antigens were isolated and characterized by precipitation in agarose gel with certain test sera. Presence of precipitating antibodies to native DNA was determined by discontinuous counter-current immunoelectrophoresis. Details of the methods employed have been reported previously (Parker, 1973).

DISEASE ACTIVITY IN SLE

This was graded from 0 to 3 in order of increasing activity as described by Gonzalez and Rothfield (1966). Nephritis in SLE was defined by the presence of at least one of the following in the absence of other explanation: RBC casts; microscopical haematuria, pyruria, and casts; proteinuria greater than $1 \mathrm{~g} / 24 \mathrm{hrs}$; or advancing renal failure (Parker, 1973).

\section{Results}

FLUORESCENT PATTERNS AND NUCLEAR

SOURCE (FIGS 1 AND 2)

Each nuclear substrate employed resulted in fluorescent patterns of acceptable clarity and reproducible titre. Use of Evans blue as a background red fluorescent stain produced striking contrast between positive (yellow-green) and negative (red) areas of

\footnotetext{
- Microbiological Associates.
}

the nucleus (Fig. 2). Background staining was particularly useful in appreciating presence of lowtitred anti-nucleolar antibodies. Patterns observed with mouse liver were essentially the same as those observed with mouse kidney. No organ-specific antinuclear antibodies were noted in SLE that reacted preferentially with mouse kidney nuclei. Patterns obtained using cultured cells were superior to those obtained using the cryostat sections of either mouse liver or kidney. Variation of pattern from cell to cell occurred when using mouse liver or kidney and was particularly evident in those serum samples giving a peripheral fluorescent pattern, due to nuclear sectioning at various levels. In contrast, peripheral staining was quite uniform from cell to cell when cultured Vero cells were used as nuclear substrate. Sera containing antibodies reactive with nucleoli produced clearly evident staining of the one or two nucleoli present in Vero cells but produced a 'large speckled' appearance in mouse liver or kidney due to the presence of the 2 to 6 nucleoli present in each nucleus. Two distinct types of true speckled patterns were apparent using Vero cells as nuclear source; these could not be differentiated using cryostat-sectioned tissues. Speckled pattern A was characterized by the presence of discrete speckles and an entirely negative nucleolus. In contrast, speckled pattern B was characterized by a reticular network with staining at the periphery of the nucleolus. Fluorescence was abolished by prior incubation of the cell coverslip with deoxyribonuclease in the case of speckled pattern $B$.

\section{ANA AND IMMUNOGLOBULIN CLASS}

Antibodies producing the nucleolar pattern of fluorescence were always present in highest titre as IgG antibodies. Speckled patterns in SLE, scleroderma, and MCTD (type A) also occurred in highest titres as IgG antibodies, whereas in rheumatoid arthritis speckled patterns (usually type B) were found more frequently ( 23 vs. 3 ) and in higher titre as IgM antibodies. $13 \%$ of patients with rheumatoid arthritis had a low-titred IgG homogeneous ANA and a moderate-titred IgM speckled ANA type B, both antibodies occurring in the same serum sample. Aside from this pattern association with rheumatoid arthritis, determination of IgM antinuclear antibodies yielded no clinically useful information. Therefore, though all sera were tested with monospecific antisera for IgG and IgM, only information concerning IgG ANA titre and patterns will be subsequently discussed, and the term 'speckled' will refer to speckled pattern $A$.

\section{PATTERN CHANGE WITH SERUM DILUTION} In $96 \%$ of patients tested fluorescent pattern was constant with serial serum dilution. In $4 \%$ the pattern 

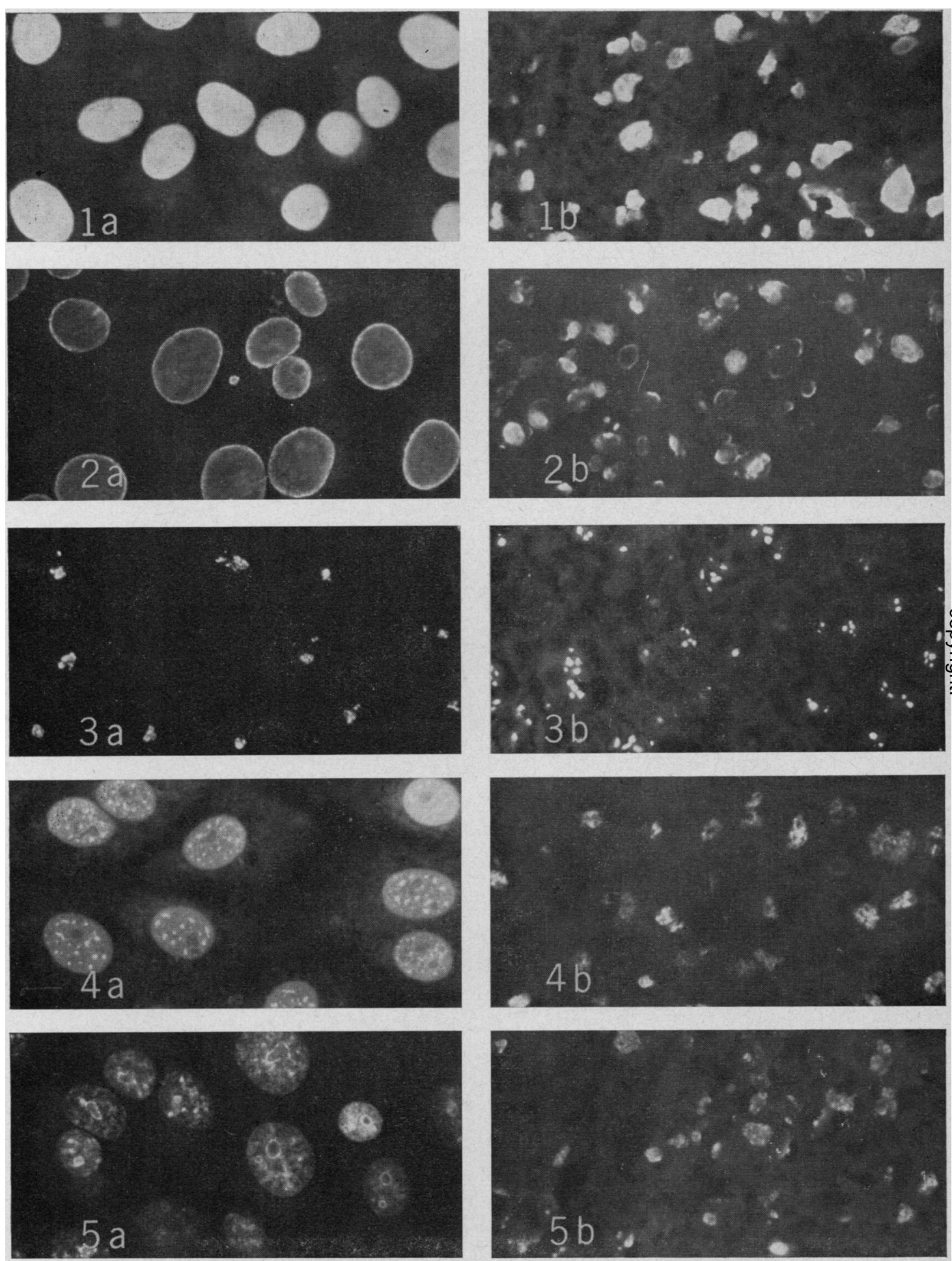

FIG. 1 Nuclear fluorescent patterns using two nuclear substrates. Number identifies pattern (1 homogeneous, 2 peripheral, 3 nucleolar, 4 speckled pattern A, 5 speckled pattern B). Letter identifies source of nuclei (a, tissue culture cells-Vero; $b$, mouse liver). $\times 440$ 

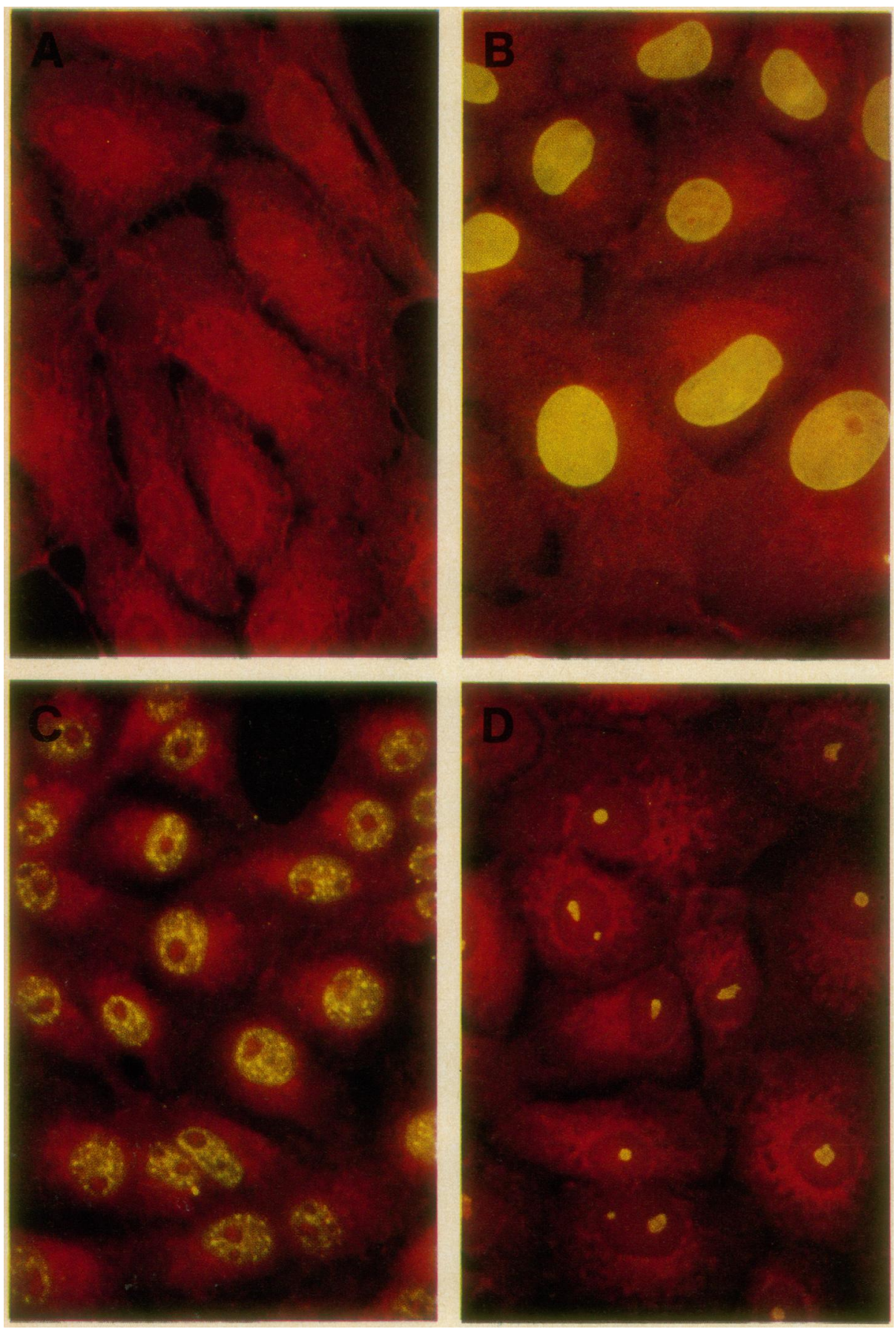

FIG. 2 Representative ANA fluorescent patterns using Evans blue as background red fluorescent stain. Yellow identifies intranuclear sites of antibody reactivity. Patterns are clearly distinguishable one from another. $A$, negative; $B$, homogeneous; $C$, speckled $(A) ; D$, nucleolar. $\times 440$, original magnification 
changed. This change was limited to sera showing a combined homogeneous/peripheral pattern where on high dilution either speckles appeared or the peripheral pattern was lost with retention of the homogeneous component. Each of these patients was found to have antibodies to one or more nuclear antigens in precipitin reactions.

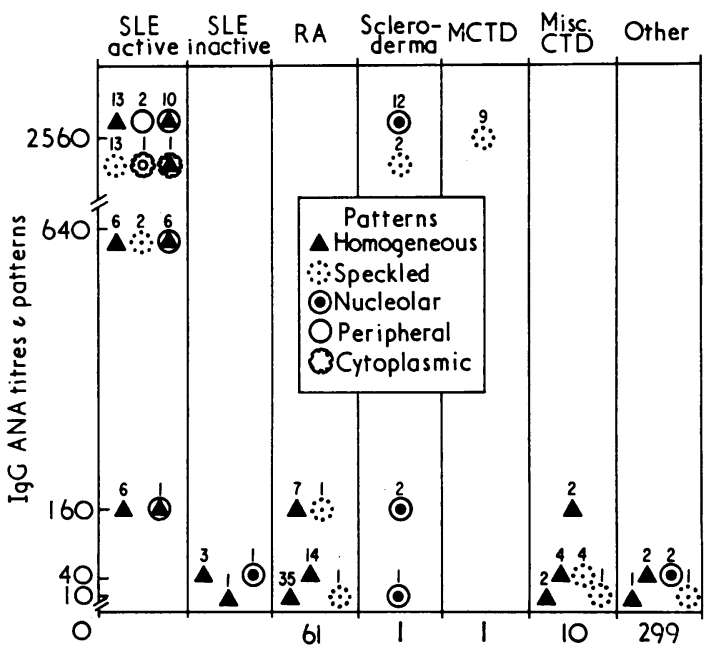

FIG. 3 IgG ANA patterns and titres in 541 patients according to disease. Symbols identify pattern of fluorescence at a particular titre. The number above each pattern symbol refers to the number of patients. Combination patterns in active SLE are indicated by appropriate combination of symbols

IgG ANA TITRES AND FLUORESCENT PATTERNS IN DISEASE

Results of combined determination of IgG ANA titre and pattern from each patient in this study are presented in Fig. 3. SLE, scleroderma, and MCTD were the only diseases associated with IgG ANA titres of $1: 640$ or higher.

\section{$S L E$}

IgG ANA were detected in all patients. In each instance IgG ANA titre was greater than IgM ANA titre. All patterns of fluorescence were noted (though in only one instance a nucleolar pattern, and that in a patient with inactive disease). Peripheral and combined homogeneous and peripheral patterns were seen exclusively in patients with active SLE and in no other illness tested. Furthermore, a strictly homogeneous pattern in titre of $1: 640$ or greater was also seen exclusively in SLE. The peripheral or peripheral/homogeneous pattern was frequently associated with nephritis $(85 \%)$ and presence of precipitating DNA antibodies $(60 \%)$. Myositis was not a feature in these SLE patients; Raynaud's phenomenon was found in one patient $(5 \%)$. Speckled patterns in SLE were less often associated with nephritis $(47 \%)$ and presence of DNA antibodies $(7 \%)$, but were more often associated with myositis $(47 \%)$ and Raynaud's phenomenon (33\%). Serositis, fever, and low serum complement were features of SLE patients with both speckled and peripheral, or combination peripheral and homogeneous, patterns. From Fig. 4 it is seen that disease activity generally paralleled ANA titres regardless of the pattern observed, with highest titres being seen in untreated patients with multisystem involvement and fever. Each SLE patient with a speckled pattern ANA had precipitating antibodies to one or more salineextractable nuclear antigens (Parker, 1973).

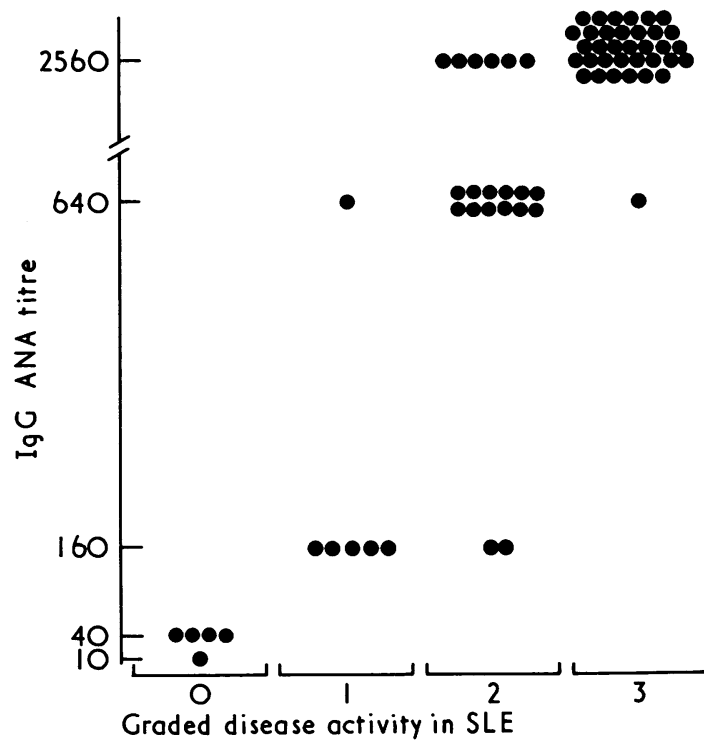

FIG. 4 IgG ANA titre according to disease activity in SLE. Each dot represents one patient

MCTD

Nine of the ten patients with MCTD had ANA. Each seropositive patient had an ANA titre of 2,560 (highest tested), a speckled ANA pattern (indistinguishable from that seen in some patients with SLE and in two patients with scleroderma), and precipitating antibodies to a specific ribonucleoprotein. Clinical characteristics of the MCTD patients studied here have been reported previously (Parker, 1973) and are essentially in agreement with the findings of Sharp and others (1972).

\section{Scleroderma}

Seventeen patients with scleroderma had antinuclear antibodies. Fourteen of these had ANA titre of 2,560, 
a titre comparable to that seen in active SLE and in MCTD. Two patients had a speckled pattern. The seronegative patient had nonprogressive illness of long duration. Fifteen patients had a nucleolar (large speckled) pattern, a pattern not found in active SLE or in MCTD. Highest titred ANA in scleroderma were found in those patients with recent onset of disease with rapid progression. In two cases nucleolar antibodies were present before a clinical diagnosis of scleroderma became evident. One of the two patients with speckled ANA had two features suggestive of MCTD including haemolytic anaemia and a prior episode of myositis. The other had persistently raised muscle enzymes but without weakness.

\section{Rheumatoid arthritis}

ANA were present in $49 \%$ of 119 patients. When IgG ANA titres in rheumatoid arthritis are compared to those seen in active SLE (Fig. 3), it is seen that all but seven active SLE patients had titres higher than the highest seen in rheumatoid arthritis, a difference that is highly statistically significant $(P<0.001)$. In contrast, SLE patients with inactive disease could not be identified by IgG ANA titre from patients with rheumatoid arthritis or with a variety of other illnesses. Two patients with rheumatoid arthritis and ANA titre of 1:160 had features suggestive of SLE. One had recurrent malar rash after sun exposure and the other persistent leucopenia without other findings to suggest Felty's syndrome. Two rheumatoid arthritis patients had weakly positive LE cell preparations without clinical evidence of SLE. Presence of ANA in RA did not correlate with degree of clinical activity, sedimentation rate, duration of illness, sex, or age. In one patient whose course was followed from active disease to remission on gold therapy, ANA persisted at the same titre throughout the course.

\section{Other illnesses}

Of the 23 patients with polymyositis and miscellaneous connective tissue diseases thought possibly to involve immune mechanisms, 12 patients had ANA titre of $1: 10$ to $1: 160$. In a control group of 143 patients with a variety of nonconnective tissue disease, 6 (4\%) had ANA all in low titre. There was nothing specific in pattern or titre of ANA that allowed differentiation of these illnesses based solely on the determination of ANA titre and pattern. ANA were not noted in the normal control group.

\section{Discussion}

Previous reports have indicated an association between nuclear fluorescent patterns and antibodies to specific nuclear components. Homogeneous patterns are associated with antibodies to deoxyribonucleoprotein (Lachmann and Kunkel, 1961), the antibodies responsible for the LE cell phenomenon. Peripheral patterns are associated with antibodies to DNA (Casals, Friou, and Teague, 1963). The finding that peripheral patterns are not always abolished by prior incubation of test serum with DNA may be explained by the findings of Tan (1967) that antibodies to a soluble nucleoprotein (sNP) also may be associated with the peripheral staining pattern. The data of Rothfield and Stollar (1967) supported their conclusion that DNA within a DNA-protein complex might be the nuclear antigen responsible for the peripheral pattern. The antigens responsible for the speckled and nucleolar patterns of fluorescence have not been clearly defined. Nucleolar antibodies are most likely reactive with a precursor ribonucleoprotein (Beck, 1963). Speckled patterns of fluorescence have been associated with antibodies to a deoxyribonuclease-, ribonuclease-, and trypsin-resistant nuclear antigen containing carbohydrate termed the Sm antigen by Tan and Kunkel (1966). Sharp and others (1972), Mattioli and Reichlin (1971), and Northway and Tan (1972) have shown that antibodies reactive with ribonucleoprotein present in extracts of nuclei also produce a speckled pattern of fluorescence in MCTD, SLE, and scleroderma.

Several reports have commented on a diagnostically significant association between patterns of nuclear fluorescence and specific rheumatic diseases. For example, peripheral patterns were reported to be unique to SLE (Gonzalez and Rothfield, 1966) and nucleolar patterns (especially in high titre) to be characteristic in scleroderma (Ritchie, 1970). Other reports have questioned the specificity of peripheral patterns for SLE (Dorsch and others, 1969) and indicated that speckled patterns are more often associated with scleroderma than are nucleolar patterns (Rothfield and Rodnan, 1968). Ritchie (1967) reports that titration of ANA may be helpful in distinguishing patients with SLE and scleroderma (high titre) from patients with other rheumatic diseases (low titre) regardless of fluorescent pattern.

The present study surveyed a group of 541 patients with respect to both ANA pattern and titre. Use of tissue cultured cells as the nuclear source resulted in sharper patterns than are obtainable using cryostat sectioned tissues. Only information concerning IgG ANA titres and patterns has been presented in detail since it was found that IgM ANA titres and patterns were of limited clinical value.

Combined determination of both IgG ANA titre and pattern contributed greater disease specificity to the test than did either determination alone. Specifically, identification of high ANA titre served to separate patients with (1) active SLE, a majority of patients with (2) scleroderma, and (3) MCTD from patients with other connective tissue diseases with ANA (low titred). When combined with determination of fluorescent pattern, the three groups with 
high titres could then be differentiated further serologically as well as clinically. Thus, only patients with clinical scleroderma had high titred nucleolar antibodies, and only SLE patients had high titred homogeneous or peripheral patterns, separately or in combination. Those SLE and scleroderma patients with speckled ANA patterns could be separated from MCTD patients on clinical grounds but they could not be separated on serological grounds simply by finding a speckled pattern ANA test in high titre. However, of those ten patients with high titred speckled patterns which disappeared upon incubation of the nuclear source with ribonuclease, nine had MCTD (one had scleroderma clinically); furthermore, of those patients with high titred speckled patterns completely or partially resistant to ribonuclease, all but one had SLE clinically.

In SLE patients, both clinical and laboratory differences were noted between those groups with peripheral patterns of fluorescence and those with speckled patterns. Peripheral patterns were associated with nephritis and DNA antibodies as previously noted by Rothfield and Stollar (1967), whereas speckled patterns were associated less often with nephritis and DNA antibodies but more often with Raynaud's phenomenon and a mild and nonprogressive myositis, both uncommon findings in patients with peripheral or homogeneous patterns. Multisystem disease with serositis and fever was seen in both groups. In MCTD renal disease was absent, and clinical features as previously described (Parker, 1973) were similar to those reported earlier by Sharp and others (1972).

Although simultaneous determination of IgG ANA titre and pattern was more useful than either alone, two patterns were sufficiently frequently associated with particular diseases to be of evident significance by themselves: all of the twenty patients with IgG peripheral patterns were SLE patients; fifteen of eighteen patients with nucleolar pattern had scleroderma. When present in high titre, a nucleolar pattern appeared to be as specific a confirmatory laboratory test in scleroderma as is currently available. The specificity of the nucleolar pattern is in agreement with the findings of Ritchie (1970). Though these results are seemingly contrary to the report of Rothfield and Rodnan (1968), which associates scleroderma with speckled pattern ANA, perhaps they are not. When mouse liver is used as nuclear substrate, nucleolar antibody-containing serum does indeed yield a 'large speckled' pattern (Fig. 1), due to the presence of multiple nucleoli in mouse liver nuclei (Busch and Smetana, 1970). Also differences in patient selection may occasionally be reflected, due to clinical overlap between scleroderma and MCTD. The latter illness is uniformly associated with a speckled pattern ANA (Sharp and others, 1972; Parker, 1973). Specificity of the peripheral pattern for SLE is in agreement with the report of Gonzalez and Rothfield (1966), but contrary to the reports of Dorsch and others (1969) and Burnham, Fine, and Neblett (1966). Variations in reports may sometimes be due to differences in technique. In the report of Burnham and others (1966), for example, the fluorescent conjugate used was polyspecific with antibodies to IgG, IgM, and IgA. In the present study using antisera monospecific to IgG and IgM, respectively, peripheral patterns were observed in five patients with rheumatoid arthritis in low titre, but in each case they were produced by IgM antibodies. It is apparent, therefore, that unless either monospecific antiserum to IgG is used in the determination of patterns or IgM is completely inactivated from serum, peripheral patterns cannot be considered specific for SLE. The problem is further resolved by the finding here that with rare exception IgG ANA titres are much higher in active SLE than in rheumatoid arthritis regardless of pattern seen. Demonstration of antibodies to native DNA can also contribute to the serological characterization of SLE patients (Parker, 1973; Kredich, Skyler, and Foote, 1973).

Tan (1967) and Dorsch and others (1969) have pointed out that a given pattern may change with serial dilution of serum. In the present study these changes were infrequent and indicated that multiple antigen-antibody systems were involved in producing these patterns. Each such patient studied here had SLE.

In any survey such as the present one, patients included in the study must meet defined clinical criteria in order to permit evaluation of the serological data compiled. Once this is done, however, the correlations observed may be used in looking at other patients who do not as yet meet such clinical criteria, in an attempt to understand at an earlier stage the direction of clinical events.

\section{Summary}

Combined determination of IgG antinuclear antibody (ANA) titre and nuclear fluorescent pattern was made on 541 serum samples. Use of cultured cells as nuclear substrate allowed clearer definition of ANA patterns than was possible through use of cryostat sectioned tissues. Most patients with active SLE, scleroderma, and MCTD had IgG ANA titres significantly higher than those found in other connective tissue diseases. When such high titres were found, fluorescent patterns then provided a serological basis for differentiation of these three diseases. The combined determination of titre and pattern offered more specific confirmatory serological data than did either determination alone. Neither test was found to be of value in the characterization of the other connective tissue diseases studied. 


\section{References}

Alexander, W. M. R., Bremner, J. M., ANd Duthie, J. J. R. (1960) Ann. rheum. Dis., 19, 338 (Incidence of the antinuclear factor in human sera)

BECK, J. S. (1963) Scot. med.J., 8, 373 (Autoantibodies to cell nuclei)

-, Anderson, J. S., Bloch, K. J., Buchanan, W. W., AND Bunin, J. J. (1965) Ann. rheum. Dis., 24, 16 (Antinuclear and precipitating autoantibodies in Sjogren's syndrome)

Burnham, T. K., Fine, G., AND Neblett, T. R. (1966) Ann. intern. Med., 65, 9 (The immunofluorescent tumor imprint technique II. The frequency of anti-nuclear factors in connective tissue diseases and dermatoses)

Busch, H., AND Smetana, K. (1970) In 'The Nucleolus', p. 137. Academic Press, New York

Casals, S. P., Friou, G. J., AND MYers, L. L. (1964) Arthr. and Rheum., 7, 379 (Significance of antibody to DNA in systemic lupus erythematosus)

- - , AND TEAGUE, P. O. (1963) J. Lab. clin. Med., 62, 625 (Specific nuclear reaction pattern of antibody to DNA in lupus erythematosus sera)

Cohen, A. S., Reynolds, W. E., Franklin, E. C., Kulka, J. P., Ropes, M. W., Shulman, L. E., and Wallace, S. L. (1971) Bull. rheum. Dis., 21, 643 (Preliminary criteria for the classification of systemic lupus erythematosus)

Dorsch, C. A., GibBs, C. B., Stevens, M. B., ANd Shulman, L. E. (1969) Ann. rheum. Dis., 28, 313 (Significance of nuclear immunofluorescent patterns)

Friou, G. J. (1957) J. clin. Invest., 36, 890 (Clinical application of lupus serum-nucleoprotein reaction using the fluorescent antibody technique)

-(1967) Arthr. and Rheum., 10, 151 (Antinuclear antibodies: diagnostic significance and methods)

Gonzalez, E. N., AND Rothfield, N. F. (1966) New Engl. J. Med., 274, 1333 (Immunoglobulin class and pattern of nuclear fluorescence in systemic lupus erythematosus)

HoLBOROW, E. J., WeIR, D. M., AND JoHNSON, G. D. (1957) Brit. med. J., 2, 732 (A serum factor in lupus erythematosus with affinity for tissue nuclei)

KREDICH, N. M., SKYLER, J. S., AND FOOTE, L. J. (1973) Arch. intern. Med., 131, 639 (Antibodies to native DNA in systemic lupus erythematosus-a technique of rapid and quantitative determination)

LACHMANN, P. J., AND KUNKeL, H. G. (1961) Lancet, 2, 436 (Correlation of antinuclear antibodies and nuclear staining patterns)

MATTIOLI, M., AND ReICHLIN, M. (1971) J. Immunol., 107, 1281 (Characterization of a soluble nuclear ribonucleoprotein antigen reactive with SLE sera)

NorthWAY, J. D., AND TAN, E. M. (1972) Clin. Immunol. Immunopath., 1, 140 (Differentiation of antinuclear antibodies giving speckled staining patterns in immunofluorescence)

PARKER, M. D. (1973) J. Lab. clin. Med., 82, 769 (Ribonucleoprotein antibodies: frequency and clinical significance in systemic lupus erythematosus, scleroderma, and mixed connective tissue disease)

Pincus, T., Schur, P. H., Rose, J. A., Decker, J. L., ANd Talal, N. (1969) New Engl. J. Med., 281, 701 (Measurement of serum DNA-binding activity in systemic lupus erythematosus)

RrTCHI, R. F. (1967) Arthr. and Rheum., 10, 544 (The clinical significance of titred antinuclear antibodies)

(1970) New Engl.J. Med., 282, 1174 (Antinucleolar antibodies: their frequency and diagnostic association)

Ropes, M. W., BennetT, G. A., CoBB, S., JACOX, R., AND JessAR, R. A. (1958) Bull. rheum. Dis., 9, 175 (1958 revision of diagnostic criteria for rheumatoid arthritis)

Rothfield, N. F., Frangione, B., and Frankin, E. C. (1965) J. clin. Invest., 44, 62 (Slowly sedimenting mercaptoethanol-resistant antinuclear factors related antigenically to $\mathbf{M}$ immunoglobulins (gamma $1 \mathrm{M}$ globulin) in patients with systemic lupus erythematosus))

- AND RoDNAN, G. P. (1968) Arthr. and Rheum., 11, 607 (Serum antinuclear antibodies in progressive systemic sclerosis (scleroderma))

-, AND STOLLAR, B. D. (1967) J. clin. Invest., 46, 1785 (The relation of immunoglobulin class, pattern of antinuclear antibody, and complement-fixing antibodies to DNA in sera from patients with systemic lupus erythematosus)

Ruderman, M., Miller, L. M., and Pinals, R. S. (1968) Arthr. and Rheum., 11, 377 (Clinical and serologic observations on 27 patients with Felty's syndrome)

SCHUR, P. H. (1970) New Engl.J. Med., 282, 1205 (A.N.A.)

Sharp, G. C., Irvin, W. S., TAN, E. M., Gould, R. G., and Holman, H. R. (1972) Amer. J. Med., 52,148 (Mixed connective tissue disease-an apparently distinct rheumatic disease syndrome associated with a specific antibody to an extractable nuclear antigen (ENA))

TAN, E. M. (1967) J. Lab. clin. Med., 70, 800 (Relationship of nuclear staining patterns with precipitating antibodies in systemic lupus erythematosus)

-, AND KUNKEL, H. G. (1966) J. Immunol., 96, 464 (Characteristics of a soluble nuclear antigen precipitating with sera of patients with systemic lupus erythematosus) 